\title{
Breaking the Misconception about Alcohol: Editorial Go with the Light, Out of the Monochrome
}

\author{
Seung-Won Oh
}

Department of Family Medicine, Seoul National University Hospital Healthcare System Gangnam Center, Seoul, Korea

One of the best-known beliefs regarding alcohol is that moderate alcohol consumption is beneficial for health. In the West, at the beginning of the 1990s, the French population was observed to have a low rate of cardiovascular disease relative to their saturated fat consumption; this was dubbed the 'French paradox. ${ }^{1)}$ As moderate wine consumption received attention as a cause of this phenomenon, wine consumption has increased in popularity. Since then, questions have been raised as to whether the 'French paradox' actually exists. Even if this phenomenon does exist, the current consensus is that the fundamental reason for this is the overall difference in dietary habits rather than in consumption of a specific food item, such as wine. However, this has had a significant impact in reinforcing the belief that alcohol confers health benefits. In Korea, a similar belief had already existed since a much earlier date. This can be seen from the use of the word 'yakju' (medicinal alcohol), which has traditionally been used to refer to alcohol in a respectable manner. This word may have originally referred to alcohol produced from herbs; however, it is now used to refer to all alcohol irrespective of type. The term includes the Chinese character 'yak' (medicine), which has contributed to the perpetuation of the belief that alcohol confers health benefits. We have often encountered people who believe that alcohol is good for blood circulation.

Numerous epidemiological studies have reported that drinking one or two glasses of alcohol per day can reduce the risk of cardiovascular disease. ${ }^{2)}$ The proposed mechanism for this positive effect relates to the fact that moderate alcohol consumption increases high-density lipoprotein cholesterol levels and suppresses platelet aggregation. Based on these findings, the health benefits of alcohol consumption can be viewed as a scientific fact rather than a general belief. However, caution should be exercised in the application of this scientific fact as, compared to westerners, a significant proportion of Koreans are at increased risk of ill-health and are unlikely to see even modest benefits in terms of cardiovascular disease from a moderate alcohol consumption.

Aldehyde dehydrogenase in mitochondria $(\mathrm{ALDH} 2)$ is the main enzyme involved in the metabolism of acetaldehyde, a toxin produced during alcohol metabolism. When a point mutation occurs in its corresponding gene, an inactive form of ALDH2 is produced. This type of mutation is rare in Westerners; however, $36 \%$ to $52 \%$ of Asians express the inactive form of ALDH2. ${ }^{3,4)}$ In these people with inactive form of ALDH2, acetaldehyde accumulates following alcohol consumption, resulting in flushing, palpitations, nausea, and vomiting. Of these responses, flushing is the most useful in predicting whether a person carries the inactive form of ALDH2.

People who show a flushing response to alcohol (flushers), i.e., people with the inactive form of $\mathrm{ALDH} 2$, are at increased risk of liver damage and esophageal cancer. ${ }^{5)}$ In addition, differences may exist between flushers and non-flushers, in terms of the benefits of moderate alcohol consumption in relation to cardiovascular disease. In the present issue, Suh et al. ${ }^{6)}$ describe a study conducted on 1,817 Korean men and report that nonflushers consuming 4 drinks or less per week had a significantly lower risk of cardiovascular diseases than non-drinkers; however, moderate alcohol consumption did not confer any benefits to flushers. Similar to this study, the same research team previously demonstrated differences between flushers and non-flushers with respect to risk of insulin resistance, metabolic syndrome, and hyperhomocysteinemia. ${ }^{7-9)}$ The present study and other similar studies of Korean subjects are limited by the fact that they are 
cross-sectional studies and, therefore, further verification of causal relationships through longitudinal studies is required.

Korea faces a number of alcohol-related social problems. Korean drinking culture is the primary cause: offering a drink is seen as hospitable, and heavy drinking is seen as a symbol of masculinity. However, traditional beliefs regarding the health benefits of alcohol may also contribute. According to statistics from 2012, the percentage of Korean men and women (age $\geq 19$ years) who drink once a month or more was $73.5 \%$ and $42.9 \%$, respectively. ${ }^{10)}$ This implies that a significant number of flushers consume alcohol in spite of the potential risks associated with drinking. If genetic differences, including the flushing response, are not taken into consideration when evaluating the effects of alcohol on health, the notion that moderate drinking confers health benefits might be a misconception. In the future, to dissuade inaccurate beliefs, a person's complexion may need to be checked under the light before offering them a drink.

\section{CONFLICT OF INTEREST}

No potential conflict of interest relevant to this article was reported.

\section{REFERENCES}

1. Renaud S, de Lorgeril M. Wine, alcohol, platelets, and the French paradox for coronary heart disease. Lancet 1992;339:1523-6.

2. O’Keefe JH, Bybee KA, Lavie CJ. Alcohol and cardiovascular health: the razor-sharp double-edged sword. J Am Coll Cardiol 2007;50:1009-14.
3. Agarwal DP, Harada S, Goedde HW. Racial differences in biological sensitivity to ethanol: the role of alcohol dehydrogenase and aldehyde dehydrogenase isozymes. Alcohol Clin Exp Res 1981;5:12-6.

4. Mizoi Y, Tatsuno Y, Adachi J, Kogame M, Fukunaga T, Fujiwara S, et al. Alcohol sensitivity related to polymorphism of alcohol-metabolizing enzymes in Japanese. Pharmacol Biochem Behav 1983;18 Suppl 1:127-33.

5. Yokoyama T, Yokoyama A, Kato H, Tsujinaka T, Muto M, Omori T, et al. Alcohol flushing, alcohol and aldehyde dehydrogenase genotypes, and risk for esophageal squamous cell carcinoma in Japanese men. Cancer Epidemiol Biomarkers Prev 2003; 12(11 Pt 1):1227-33.

6. Suh HS, Kim JS, Kim SS, Jung JG, Yoon SJ, Ahn JB. Influence of the flushing response in the relationship between alcohol consumption and cardiovascular disease risk. Korean J Fam Med 2014;35:295-302.

7. Jung JG, Kim JS, Oh MK. The role of the flushing response in the relationship between alcohol consumption and insulin resistance. Alcohol Clin Exp Res 2010;34:1699-704.

8. Jung JG, Kim JS, Yoon SJ, Oh MK. Relationships among alcohol consumption, facial flushing response, and metabolic syndrome in healthy men. Ann Epidemiol 2012;22:480-6.

9. Kim EC, Kim JS, Jung JG, Kim SS, Yoon SJ, Ryu JS. Effect of alcohol consumption on risk of hyperhomocysteinemia based on alcohol-related facial flushing response. Korean J Fam Med 2013;34:250-7.

10. Ministry of Health and Welfare, Korea Centers for Disease Control and Prevention. Korea health statistics 2012: Korea National Health and Nutrition Examination Survey (KNHANES V-3). Cheongju: Korea Centers for Disease Control and Prevention; 2013. 